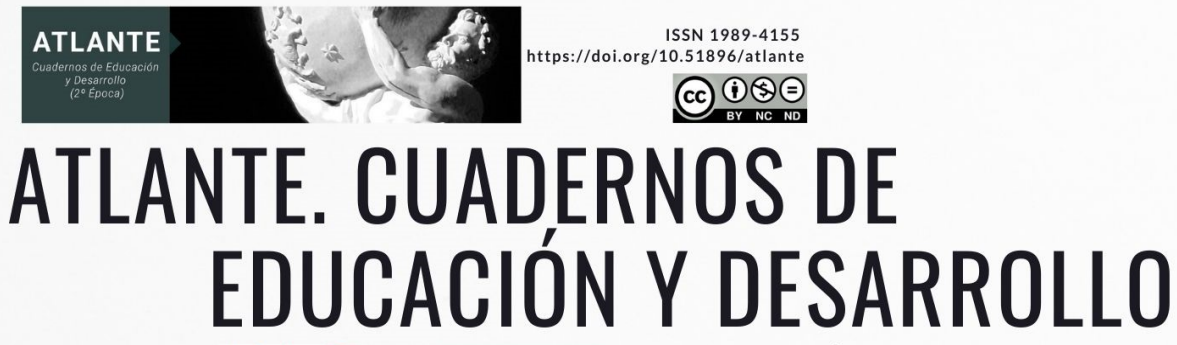

latindex @idEAS EconPapers ODialnet MIAR İDICEs

\title{
LOS ESTILOS DE APRENDIZAJE Y LOS ESTILOS DE ENSEÑANZA EN LA CLASE DE LENGUAS EXTRANJERAS
}

\author{
MSc. Annia Rosales Romero. \\ Universidad de Guantánamo. Guantánamo, Cuba. \\ ORCID: 0000-0002-6116-9243 \\ rosalesromeroannia@gmail.com
}

Para citar este artículo puede utilizar el siguiente formato:

Annia Rosales Romero: "Los estilos de aprendizaje y los estilos de enseñanza en la clase de lenguas extranjeras", Revista Atlante: Cuadernos de Educación y Desarrollo, ISSN: 1989-4155 (vol 13, № 7 octubre-diciembre 2021, pp. 65-69). En línea:

https://doi.org/10.51896/atlante/HENS7314

\section{RESUMEN}

Uno de los temas más investigados en el mundo hoy en día es relacionado con los estilos de aprendizaje; teniendo en cuenta que los estilos de aprendizaje no son más que las diferentes vías o canales que utilizamos para percibir, analizar, decodificar e interiorizar la información. En el presente artículo se hace referencia a los diferentes estilos de aprendizaje de manera general y en particular al modelo de Programación Neurolingüística (PNL). Se presentan los diferentes canales de percepción de los estudiantes y profesores determinando el más predominante, y la relación que existe entre los estilos de aprendizaje de los profesores y los estilos de enseñanza que utilizan para impartir el contenido. Se presentan los resultados de unos instrumentos aplicados para determinar esta relación y analizar de qué manera influye en la calidad del aprendizaje de los estudiantes.

Palabras claves: estilos, aprendizaje, enseñanza, estudiantes, profesores.

\section{THE LEARNING STYLES AND THE TEACHING STYLES IN THE FOREIGN LANGUAGE LESSON}

\section{ABSTRACT}

One of the most researched themes all over the world nowadays is related to the learning styles; taking into account that learning styles are the different ways or receptive channels that we use to perceive, analyse, decode, and internalize information. In this article the authoresses make reference to the learning styles in general and particularly to the Neuro Linguistic Programming (NLP). It is presented the different receptive channels that the students and professors have determining the predominant one, as well as the relationship between the learning styles of the professors and the learning strategies they use at the time of teaching a content. It is also presented the results of some 
instruments applied to determine this relationship and analyse in which way this relationship affects in the students ' learning quality.

Key words: styles, learning, teaching, students, professors.

\section{INTRODUCCIÓN}

Por años, los maestros han estado preocupados más por las técnicas de enseñanza a utilizar que por los procesos de aprendizaje, considerando que una buena técnica de enseñanza es aquella que concuerda con las técnicas de aprendizaje de los estudiantes. De todos modos, esta situación es algo controversial, ya que la enseñanza y el aprendizaje son dos procesos distintos. La enseñanza es una actividad observable llevada a cabo por el maestro hacia un estudiante o grupo de estudiantes. Por otra parte, el aprendizaje es algo que ocurre normalmente en las personas a través de sus vidas, y no es necesaria la presencia de un maestro. Nadie hasta ahora ha podido ver cómo ocurre ese proceso porque es una actividad invisible. Todo esto significa que tanto la enseñanza como el aprendizaje necesitan del uso de vías apropiadas para llevar a cabo el proceso cognitivo.

En los últimos años los estilos de aprendizaje han sido una de las áreas más investigadas en el mundo, se pueden citar: (Naiman,1978), (Robin, 1975), (Ellis, 1985), (Sinclair, 1989); (Willing, 1989), (Nunan, 1989), (O’Malley, Chamot, Stewner, Manzanares, Küpper y Russo, 1985), (Cavelluci, Alonso y Gallego, 2002), J. Fita Barros y Natalicio Luemba, Kolb, Senna, Mirtha Manzano Díaz, entre otros.

Para comprender mejor lo que estilos de aprendizaje significa en la enseñanza de una Lengua Extranjera, es necesario hacer un análisis detallado de lo que entendemos por estilos de aprendizaje, veamos los criterios de algunos de estos autores:

Alonso (2002), considera que las personas aprenden de forma distinta, tanto niños como adultos, personas de un país u otro, de una cultura u otra. Que todos prefieren un ambiente, unos métodos, un grado de estructura.

En 2010, Senna señala que el proceso de aprendizaje es extremadamente complejo, no se restringe a la adquisición de respuestas o conocimientos. Varios estudios fueron propuestos sobre el tema y diversas teorías surgieron a partir de ahí. Un punto en común de todos esos estudios es la indicación de que cada individuo tendría un ritmo y una forma característica de aprender, o sea, los individuos no aprenderían de una forma homogénea.

Kolb (2002), destacó que la forma de aprender es fruto de la herencia que traemos, de las experiencias anteriores y de las exigencias actuales del ambiente.

Como se puede apreciar varias son las concepciones con respecto a los estilos de aprendizaje, pero todas, de una forma $u$ otra, van dirigidas a las formas, métodos que utiliza la persona para interiorizar y decodificar información. Los estilos de aprendizaje se refieren a las vías o métodos que cada individuo prefiere para aprender, por lo que el estilo de aprendizaje puede resultar 
de las variables de la personalidad incluyendo la estructuración psicológica y cognitiva, el contexto sociocultural y la experiencia educacional que presenta cada individuo.

En la enseñanza de una lengua extranjera, los estudiantes necesitan utilizar diferentes vías para trabajar las reglas de vocabulario y de gramática, así como otros aspectos relacionados con el idioma para poder comunicarse a través de él.

A esto es lo que llamamos estrategias de aprendizaje o estilos de aprendizaje, que no es más que los procesos mentales o vías que los estudiantes utilizan para aprender y emplear una lengua extranjera.

Hoy en día se consideran varias teorías y modelos relacionados con los estilos de aprendizaje en el presente artículo se va a utilizar uno de ellos, el Modelo de Programación Neurolingüística de Bandler y Grinder.

El nombre de PNL (en inglés NLP = Neuro Linguistic Programming) se refiere a tres aspectos básicos de la experencia humana: Neuro, Linguistic, Programming.

Este modelo, según Manzano (2007), desarrolla el criterio neurolingüístico, el cual considera que la vía de entrada de la información a través de nuestros órganos de los sentidos (ojo, oído, cuerpo; o, si se quiere, el sistema de representación visual, auditivo, kinestésico) resulta fundamental en las preferencias de quien aprende o enseña.

La PNL tiene en cuenta tres canales de recepción de información; el visual (vista), el auditivo (oído) y el kinestésico (todo el cuerpo). Es muy importante que el profesor conozca los canales de recepción de sus alumnos porque así sabe que estrategias de enseñanza utilizar. Para determinar estos canales hay varias técnicas o test que se pueden utilizar. En este trabajo se tuvo en cuenta un cuestionario para poder determinar los canales de percepción de los estudiantes.

El cuestionario fue aplicado a 15 estudiantes de tercer año de la carrera de Lenguas Extranjeras y a 6 profesores que integran el colectivo pedagógico. Se observaron dos clases para determinar si las estrategias de enseñanza de los profesores se correspondían con los estilos de aprendizaje de los estudiantes.

A través de la aplicación de estos instrumentos y cuestionarios se pudo constatar que los profesores no conocen los canales de percepción predominantes en los estudiantes, por lo que no lo tienen en cuenta a la hora de planificar e impartir las clases, las mismas se parecen más a los estilos de aprendizaje de los profesores y no al de los estudiantes, es decir que el profesor tiene en cuenta más su canal de percepción para impartir clases que el de los estudiantes, por lo que la comunicación no fluye adecuadamente y se crean lagunas en el aprendizaje afectando la calidad del mismo.

El canal de percepción predominante en los estudiantes, de acuerdo al modelo de PNL, es el kinestésico, 11 estudiantes de 15 utilizan este canal (73.3\%), un estudiante utiliza el canal visual (6.6\%) y tres el canal auditivo (20\%), en el caso de los estudiantes kinestésicos, tres combinan este canal con el visual (27.3\%) y dos con el auditivo (18.2\%). De los cuatro varones que tiene el grupo 
dos son kinestésicos (13.3\%), uno es visual (6.6\%) y el otro es kinestésico visual (6.6\%); de las hembras dos estudiantes utilizan el canal auditivo (13.3\%), el resto (9) utilizan el canal kinestésico $(60 \%)$. Como se puede apreciar el canal predominante en los estudiantes es el kinestésico por lo que la manera más fácil de ellos aprender es a través de sensaciones, emociones, trabajo en equipo y haciendo.

Los profesores, tres utilizan el canal kinestésico (50\%), dos utilizan el canal auditivo (34\%) y el otro presenta un estilo visual (16\%).

Como se puede apreciar el 50 por ciento de los profesores del grupo coinciden con el canal de recepción de los estudiantes, el kinestésico. No obstante, las estrategias de enseñanza que utilizan los mismos no responden a los otros canales que utilizan los demás estudiantes. En el caso de los profesores que tienen un canal auditivo y visual no tienen muy en cuenta el canal kinestésico y esto influye mucho en el proceso de enseñanza aprendizaje porque no se logra una adecuada comunicación entre ellos, ya que las bandas de comunicación son diferentes. La eficacia en este grupo es buena por lo que todos los estudiantes están aprobados, el problema está en que más del 50 por ciento de las notas no son buenas por lo que podemos decir que el aprendizaje no es eficiente.

En las clases observadas se pudo constatar que la estrategia de enseñanza que el profesor utiliza responde más a los canales visuales y auditivos que al estilo kinestésico, por lo que no hay una correspondencia entre la estrategia de enseñanza utilizada por el profesor para impartir el contenido de sus clases y los canales de recepción predominantes en los estudiantes. Esto es debido a que el profesor no tiene conocimiento de estos canales y no cuenta con las herramientas para poder comunicarse con sus estudiantes, aunque coincidan en sus canales de recepción de información.

\section{CONCLUSIONES}

Muchas han sido las investigaciones realizadas en diferentes partes del mundo con relación a las diferentes estrategias o estilos de aprendizaje que las personas utilizan para analizar, entender, interpretar, deducir y concebir la información.

Cada persona se caracteriza según el estilo de aprendizaje que desarrolle y según sus características de la personalidad es la estrategia de enseñanza que emplea, de ahí que cada profesional pedagógico debe diagnosticar a sus estudiantes, no solo en la parte cognitiva y de la personalidad sino también en los estilos de aprendizaje para conocer, dominar y poder trabajar con ellos según su diversidad y así lograr el nivel de aprendizaje deseado.

Para poder lograr estos objetivos el profesor debe tener una herramienta que pueda utilizar en el proceso de enseñanza - aprendizaje; que le brinde los mecanismos o pasos a seguir y al mismo tiempo lo prepare profesionalmente para poder aplicarla. 


\section{REFERENCIAS}

Acosta Padrón, Rodolfo, \& José Alfonso Hernández. (2001). Didáctica Interactiva de Lenguas. La Habana: Pueblo y Educación.

Addine Fernández, Fátima, Silvia Recarey Fernández, Micaela Fuxá Lavastida, \& Sonia Fernández González. (2004). Didáctica: teoría y práctica (2da ed.). La Habana: Pueblo y Educación.

Alonso García, C. M. y Gallego, D. J. (2002). Si yo enseño bien... ¿ por qué no aprenden los niños?

La Habana: Ed. Pueblo y Educación, 1986.

Anaya Nieto, Daniel. (2005). Diagnóstico en Educación. Madrid: Sanz y Torres.

Campos Perales, Vilma.(2015) Concepción teórico metodológica. para caracterizar los perfiles de estilos de aprendizaje en estudiantes de la carrera Agronomía. Tesis de doctorado. Universidad de la Habana. La Habana, Cuba

Canale, M. Theoretical Bases of Communicative Approaches to Second Language Teaching. -- S.I.: Ed.OxfordUniversity Press, 1983.

Cangelosi, J. E. Classroom Management Strategies. -- New York: Ed. Longman, 1988.

Clark, John. Curriculum Renewal in School Foreign Language Learning. -New York: Ed.OxfordUniversity Press, 1988.

Corbett, John. (2003). An Intercultural Approach to English Language Teaching. United Kingdom: Multilingual Matters.

Council of Europe. (2012). Common European Framework of Reference for Languages: Learning, teaching, assessment. Cambridge University Press. Recuperado a partir de www.uk.cambridge.org/elt

D. Valle Lima, A. (2010). La investigación pedagógica. Otra mirada. La Habana, Cuba: Editorial Pueblo y Educación.

Dunkin, Michael J. (2004). Assessing Teachers' Effectiveness. Issues in Educational Research. Education Curtin Website.

Echizárraga Batista, Reina. (2012). Metodología para el desarrollo de la competencia discursiva desde la asignatura Práctica Integral de la Lengua Inglesa I. Tesis de Maestrìa. Universidad de Guantànamo. Guantànamo, Cuba.

Gonzáles Rey, Fernando. (1995). Comunicación, personalidad y desarrollo. La Habana: Pueblo y Educación.

Manzano, M. (2007). Estilos de aprendizaje, Estrategias de lectura y su relación con el rendimiento académico en la segunda lengua. Tesis de Doctorado en Ciencias Pedagógicas. Universidad de Ciego de Ávila, Cuba y Universidad de Granada, España.

Sampieri, R. (2014). Metodología de la Investigación 5ta Edición. Recuperado de: http://www.academia.edu/6399195/Metodologia_de_la_investigacion_5ta_Edicion_Sampier

Senna, C. (2011). Tipos de aprendizagem. Recuperado de:

http: //www.slideshare.net/elianealmeida/tiposdeaprendizagem 